\title{
Ex vivo expansion of human hematopoietic stem cells and identification of mammary gland epithelial stem cells
}

\author{
Chengcheng Zhang ${ }^{1}$ \\ ${ }^{1}$ Departments of Physiology and Developmental Biology, University of Texas Southwestern Medical Center, 5323 Harry Hines \\ Boulevard, Dallas, Texas 75390-9133, USA
}

Hematopoietic stem cells (HSCs) proliferate and differentiate to give rise to all blood and immune cells. HSCs thus form the basis of bone marrow transplantation for treating leukemia, lymphoma, and other diseases. They are also considered attractive target cells for gene therapy. However, development of these important clinical applications has been severely hampered by the difficulty of ex vivo expansion of HSCs. Numerous attempts had been tried in the last several decades with only limited success. One key to solving this problem is identification of secreted proteins that stimulate HSC expansion. As a postdoctoral fellow in Harvey Lodish's laboratory, I identified a novel cell population that supports HSC expansion- $\mathrm{CD3}^{+}$Ter $119^{-}$cells isolated from embryonic day 15 mouse fetal livers. From these cells, I isolated insulin-like growth factor 2 and a group of angiopoietin-like proteins (Angptls) and showed that these novel factors supported ex vivo expansion of HSCs. Recently, as an independent investigator, I discovered an additional new HSC supportive factor. A lack of cell-surface markers of stem cells has also hampered research. To address this issue, I have collaborated with Susan Lindquist and Robert Weinberg's laboratories. We showed that the prion protein (PrP) is a surface protein characteristic of freshly isolated bone marrow HSCs and mammary gland epithelial progenitors; HSCs isolated from PrP-/- mice are defective in serial, sustained, hematopoietic engraftment. This enabled me to carry out a systematic investigation of the cell-surface phenotype of cultured HSCs. Based on this progress, I developed a simple culture system with defined factors and without serum or feeder cells that dramatically enhanced expansion of HSCs. As measured by competitive repopulation analyses, there was a $\sim 30$-fold expansion of mouse long-term HSCs in this culture system in the presence of Angptls. The culture system was further improved by inclusion of IGFBP2; the combination of Angpt15 and IGFBP2 resulted in dramatic expansion of human HSCs (unpublished data). These novel findings represent a significant advance for the field and will allow dissection of the environmental control of the cell fates of HSCs - self-renewal, apoptosis, and differentiation. It provides direct guidance for rational design of ex vivo expansion system for HSCs.

The identification of mammary gland stem cells or progenitors (MGSC) is important for the study of normal breast development and tumorigenesis. Based on their immunophenotype, we have isolated a population of mouse mammary gland cells that are capable of forming "mammospheres" in vitro. Importantly, mammospheres are enriched for cells that regenerate an entire mammary gland upon implantation into a mammary fat pad. We also undertook cytogenetic analyses of mammosphere-forming cells, which provided preliminary insight into the genomic stability of these cells after culture. Our identification of new cell-surface markers for enriching mammosphereinitiating cells, including endoglin and prion protein, will facilitate the elucidation of the cell biology of MGSC.

Keywords: hematopoietic stem cells, mammary gland epithelial stem cells

Cell Research (2008) 18:s83. doi: 10.1038/cr.2008.173; published online 4 August 2008

Correspondence: Chengcheng (Alec) Zhang

E-mail: Alec.Zhang@UTSouthwestern.edu 